\title{
Validation of in Silico Docking Analysis of Oligophenylpropanoids to Xanthine Oxidase by Correlation with in Vitro Bioassay and Its Application to Phlorotannins
}

\author{
Sheau Ling Ho ${ }^{1}$, Sheng-Fa Tsai ${ }^{2}$, Ching-Ting Lin ${ }^{2}$, Hyeung-Rak Kim ${ }^{3}$, Shoei-Sheng Lee ${ }^{2}$, \\ ${ }^{1}$ Department of Chemical \& Materials Engineering, Chinese Culture University, Taipei, Taiwan, ROC \\ ${ }^{2}$ School of Pharmacy, College of Medicine, National Taiwan University, Taipei, Taiwan, ROC \\ ${ }^{3}$ Departments of Food and Nutrition, Pukyong National University, Busan, Korea \\ Email address: \\ hxl3@faculty.pccu.edu.tw (Sheau Ling Ho), apha8@yahoo.com.tw (Sheng-Fa T.),d994230004@ntu.edu.tw (Ching-Ting Lin), \\ hrkim@pknu.ac.kr (Hyeng-Rak K.), shoeilee@ntu.edu.tw (Shoei-Sheng Lee) \\ ${ }^{*}$ Corresponding author
}

To cite this article:

Sheau Ling Ho, Sheng-Fa Tsai, Ching-Ting Lin, Hyeung-Rak Kim, Shoei-Sheng Lee. Validation of in Silico Docking Analysis of Oligophenylpropanoids to Xanthine Oxidase by Correlation with in Vitro Bioassay and Its Application to Phlorotannins. Journal of Drug Design and Medicinal Chemistry. Vol. 6, No. 1, 2020, pp. 1-6. doi: 10.11648/j.jddmc.20200601.11

Received: February 12, 2020; Accepted: March 13, 2020; Published: March 31, 2020

\begin{abstract}
The oligo-phenylpropanoids 1-7, isolated from Hyptis rhombodes, have been found to possess potent inhibitory activity against xanthine oxidase (EC 1.2.3.2, bovine milk). To rationalize such activity, computer assisted docking of these compounds and allopurinol, a positive control, on the xanthine oxidase was undertaken in this study. The docking scores, obtained by London (trimatch)-refinement (Forcefield Affinity $\Delta G$ ) mode, showed good correlation with the $\mathrm{IC}_{50}$ values. That the compounds possessing 7 '- $Z$ configuration had much better inhibitory activity than those 7 '- $E$ isomers is well rationalized by this docking study. Virtual screening of eight phlorotannins $(8-15)$ by this refinement mode found good docking scores. The bioassay result of three available ones $(9,12,13)$ also indicated the consistency with the docking scores. While refined by Forcefield-London mode, certain inconsistency among the docking score and bioassay result was observed on either phenylpropanoid oligomers or three phlorotannins. Hence the London (trimatch)-refinement (Forcefield-Affinity $\Delta G$ ) mode is recommended for virtual screening of the related phenolics. Three phlorotannins $(11,14,15)$ were found to have better docking score than 6,6'-bieckol (12) and dieckol (13), both showing comparable inhibitory activity against xanthine oxidase to allopurinol, and thus they deserve further study. In addition, as these phlorotannins are rich in the Ecklonia genus, the common edible seaweeds such as E. cava and E. stolonifera are demonstrated to be beneficial to hyperuricemic patients.
\end{abstract}

Keywords: Xanthine Oxidase Inhibitors, Oligo-phenylpropanoids, Bioassay, Molecular Docking, Phlorotannins

\section{Introduction}

Xanthine oxidase (XO) is a molybdenum-containing hydroxylase which catalyzes the oxidation of purine substrates, hypoxanthine and xanthine, to yield uric acid and reactive oxygen species [1]. The excessive production or insufficient elimination of uric acid results in hyperuricemia, which is associated with gout [2]. XO inhibitors have been proven their efficacy in lowering blood uric acid in animal models and clinical application [3]. Thus the development of xanthine oxidase inhibitors has become one of the therapeutic approaches for treating hyperuricemia.
Allopurinol, an XO inhibitor, is the most commonly used anti-gout drug in the past decades [3]. However, some incidences caused by allopurinol have been reported, including hypersensitivity reactions, Steven's Johnson syndrome, hepatitis, nephropathy, and 6-mercaptopurine toxicity [4]. Therefore, alternatives of allopurinol with potent anti-XO efficacy and less or void of side effects are in medical need.

Our recent study found that phenylpropanoid oligomers (Figure 1), isolated from Hyptis rhombodes Mart. \& Gal. [5], possessed potent anti-XO activity, comparable to allopurinol (Table 1). That study also indicated that for geometric isomers, 
those possessing a cis-styrenyl moiety are more potent than the corresponding trans isomer (e.g. 2 vs. 1; 4 vs. 3) and for those diastereomers, that having $\left(7 " R, 8^{\prime \prime} S\right)$-configuration is more potent than the corresponding (7"S, 8"S)- isomer (e.g. 6 vs. 4); and that with a carboxyl group is more potent than its methyl ester (e.g. 4 vs. 7). The rationale of such structure and activity relationship, however, required further clarification. As the crystal structure of xanthine oxidase had been documented on the Protein Data Bank (PDB ID: 1F1Q), this current study was aimed to apply computer assisted molecular docking in clarification of this relationship. In addition, the established docking refinement mode was applied as a virtual screening tool on the related polyphenolics, phlorotannins, to find potential xanthine oxidase inhibitors.
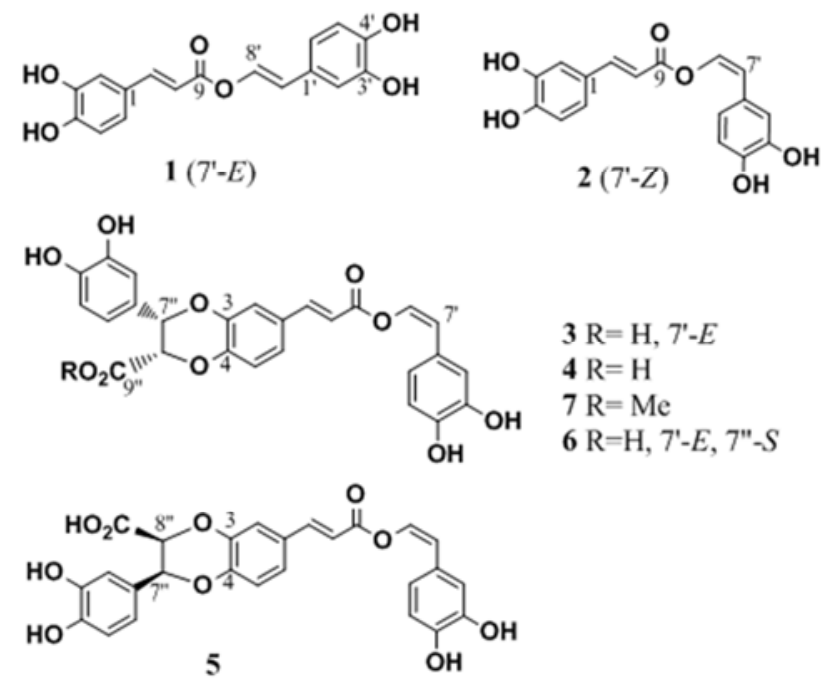

Figure 1. Phenylpropanoid oligomers isolated from Hyptis rhombodes [5]: netpetoidin A (1), netpetoidin B (2), hyprhombins $A-C$ (3-5), epihyprhombin $B$ (6), hyprhombin B methyl ester (7).

\section{Materials and Methods}

\subsection{Molecular Dynamic Simulation}

\subsubsection{Preparing the 3D Structure of Xanthine Oxidase}

The crystal structure of xanthine oxidase enzyme (XO) from bovine milk was obtained from the Protein Data Bank (PDB ID: 1F1Q). This enzyme is a homodimer. Each monomer acts independently in the catalysis process and contains a molybdopterin cofactor (Mo-pt) center, a pair of spinach ferredoxin-like clusters [2Fe-2S], and one flavin adenine dinucleotide cofactor [6]. For the molecular docking study, all small molecules except those in the binding side of $\mathrm{XO}$ were removed and the template was constructed from the remaining scaffold using the co-crystal structure of salicylate-xanthine oxidase complex as a reference [6], where the substrate binding and oxidation occur at the Mo-pt center.

Molecular docking simulations were performed using Molecular Operating Environment (MOE) [7]. Docking calculations were carried out using standard default variables of the MOE software packages. The triangle matcher algorithm was selected to dock the identified hit compounds into the Mo-pt active site [8]. The binding energy $(\Delta G)$ was estimated from the contributions of the hydrophobic and van der Waals interactions, and ionic and hydrogen bondings between XO and ligand as well as intramolecular hydrogen bondings and strains of the ligand. Binding affinity was evaluated by the binding energies (Kcal/mol) and root-mean-square deviation (RMSD) values. The RMSD value was set less than $0.6 \AA$ owing to considerable structural similarity. The ligands were docked into the groove Mo-pt binding site, and the initial model was loaded into MOE working environment ignoring water molecules and heteroatoms. The structure was put in generalized born implicit solvated environment at a constant temperature (300 $\mathrm{K})$, a constant-pH (7.0), and a salt concentration of $0.1 \mathrm{M}$. Electrostatic potential was applied to a cut-off value of $1.5 \AA$ at a dielectric value of 1.0. A non-bonded interaction with the Leonard-Jones terms was cut-off at 5.0 ̊. The dock scoring was done using London $\Delta G$ scoring function to estimate the free binding energy of the ligand from a given pose whose relaxation was refined by Forcefield refinement schemes (London or Affinity $\Delta G$ ) to enhance accuracy [9]. Rotatable bonds were allowed and the best 20 poses were retained for the binding score analysis [10]. The partial atomic charges of $\mathrm{XO}$ were assigned and energy minimization was conducted with a gradient cut-off value of $0.05 \mathrm{Kcal} / \mathrm{mol} / \AA$ to determine the geometry optimization via MMFF94x forcefield [11]. The structure of the 20 docked conformations with the lowest docking score was selected for further analysis [12].

\subsubsection{Ligands for Docking}

Seven phenylpropanoid oligomers (1-7) (Figure 1), XO inhibitors isolated from $H$. rhombodes [5], were chosen for the establishment of an appropriate refinement mode during molecular docking study. While for virtual screening against $\mathrm{XO}$, eight representative phlorotannins $(8-15)[10,11]$ were chosen. Two-dimensional (2D) structures of the selective ligands were depicted using ChemBioDraw and their three-dimensional (3D) conformations were converted into Mol files which were subjected to energy minimization and molecular dynamics using the Chem 3D pro.

\subsection{Xanthine Oxidase Inhibitory Assay}

The method used for this assay was adopted from a recent report [13]. In a 96-well plate, $\mathrm{MeOH}-\mathrm{H}_{2} \mathrm{O}(10 \mu \mathrm{L}, 1: 9$, v/v $)$ or sample in $\mathrm{MeOH}-\mathrm{H}_{2} \mathrm{O}(10 \mu \mathrm{L}, 1: 9, \mathrm{v} / \mathrm{v})$, and $2 \mathrm{mM}$ xanthine solution $(60 \mu \mathrm{L})$ was added to each well. The reaction was initiated by adding xanthine oxidase $(30 \mu \mathrm{L}, 0.2$ $\mathrm{U} / \mathrm{mL}$ ) (EC 1.2.3.2, bovine milk, Sigma). The produced uric acid was quantified by measuring the absorbance at $290 \mathrm{~nm}$ with 2 min intervals on a microplate spectrophotometer.

The inhibitory percentage against XO (\%) was calculated by the following equation: Inhibition $(\%)=\left[\begin{array}{ll}1- \\ -\end{array}\right.$ $\left.\left(\mathrm{A}_{\text {sample }} / \mathrm{A}_{\text {control }}\right)\right] \times 100$, where $\mathrm{A}_{\text {sample }}$ and $\mathrm{A}_{\text {control }}$ stand for the absorbance of the test sample and the control, respectively. Allopurinol was used as a positive control. 


\section{Results and Discussion}

\subsection{Interaction of $\mathrm{XO}$ with Oligo-phenylpropanoids 1-7}

Molecular docking of oligo-phenylpropanoids 1-7 on xanthines oxidase template was undertaken initially via London (trimatch)-refinement (Forcefield-London) mode and the results are shown in Table 1. The correlation between docking score and bioassay result $\left(\mathrm{IC}_{50}\right)$ is generally consistent except for two potent compounds, 5 and 6 , where a little disagreement was observed.

Netpetoidin A (1) and netpetoidin B (2) are geometric isomers at C-7'. The caffeoyl residue of both 1 and 2 is oriented toward the binding pocket interior of $\mathrm{XO}$, leading to the formation of H-bond with the side chain of $\operatorname{Arg} 880$ and Thr1010. Relative to 1, netpetoidin B (2) formed three additional H-bonds from the styrenyl catechol residue to Ser876, His875, and Glu879, of which Ser876 is located within and the other two close to the pocket site. Such interaction is due to different orientation of the geometrical constraint, in which 2 showed better shape complementarity as shown in Figure 2a, resulting better binding affinity $(\Delta G$ -19.32 vs. $-15.33 \mathrm{Kcal} / \mathrm{mol})$. This correlates well with the bioassay result $\left(2, \mathrm{IC}_{50} 11.7 \mu \mathrm{M} ; 1, \mathrm{IC}_{50}>159.2 \mu \mathrm{M}\right)$. Similarly, for the geometric isomers 3 and 4 , the $7^{\prime}-Z$ isomer (4) had better binding affinity than the $7^{\prime}-E$ one (3), ascribable to one additional $\mathrm{H}$-bond and better shape complementarity as depicted in Figure 2b. In addition, the residue in 3 which

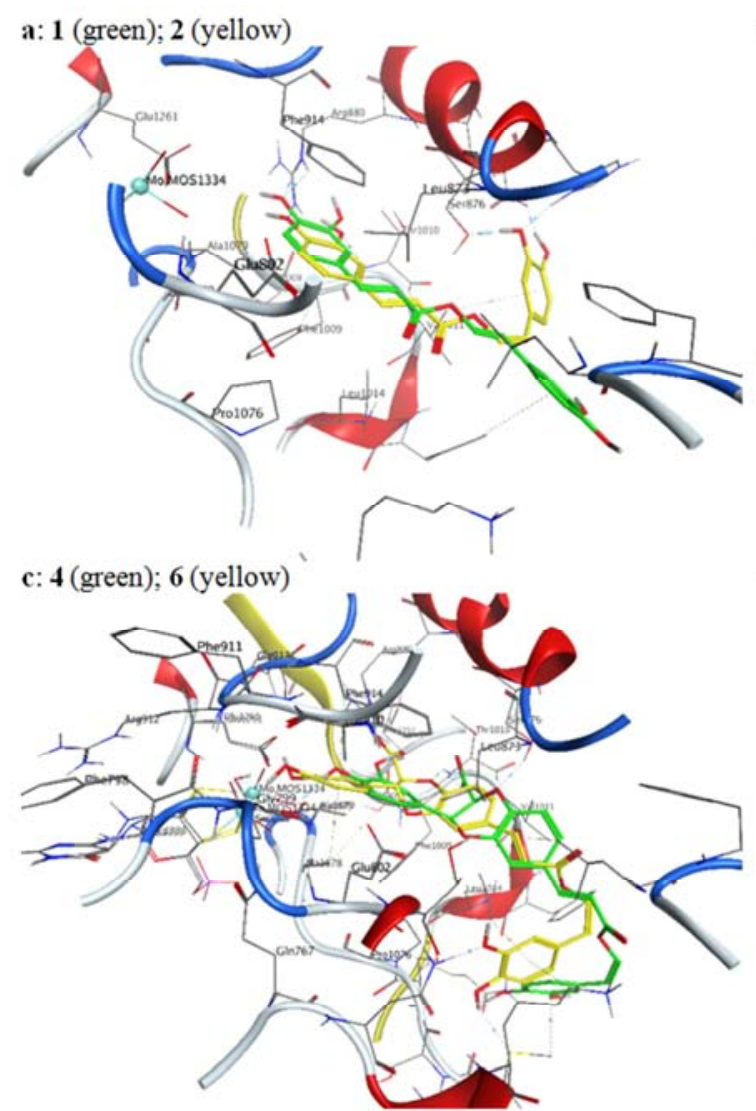

interacts with the binding pocket interior is the styrenyl catechol and that in 4 is the phenylpropenoic acid.

For the C-7" epimers 4 and 6, similar docking orientation was observed. However, the configurational difference at C-7" allowed 6 to have four additional ionic bonds between the C-9" carboxyl group and the side chain of Arg880 relative to 4 which formed only two H-bonds to Val1011 and Thr1010 as depicted in Figure 2c, accounting for better inhibitory activity of 6 ( $\mathrm{IC}_{50} 2.0$ vs. $\left.5.2 \mu \mathrm{M}\right)$. Accordingly, the contribution of the C-9" carboxyl group to the inhibitory effect against XO depends on the $\mathrm{C}-\mathrm{8}^{\prime \prime}$ configuration.

As indicated above, the C-9" carboxylic acid residue played important role in docking affinity to XO. Compound 7 , the methyl ester of 4 , should possess weaker binding affinity. Indeed, the favorite docking of 7 to $\mathrm{XO}$ showed relatively poor shape and geometric complementarity $(\Delta G-17.87$ $\mathrm{Kcal} / \mathrm{mol}$ ) within the binding site, consistent with the inhibitory activity $\left(\mathrm{IC}_{50} 30.9 \mu \mathrm{M}\right.$ vs. $\left.5.2 \mu \mathrm{M}, 4\right)$.

As for C-7"/C-8" positional isomers 5 and 6, similar docking orientation (Figure 2d) was also observed. The adjustment of the catechol and the carboxyl group to nearly overlap in both compounds makes the styrenyl catechol residue oriented quite differently. Such difference, however, affects the binding score only a little $(\Delta G-21.95$ vs. -22.07 $\mathrm{Kcal} / \mathrm{mol}$ ) since the phenylpropenoic acid residue dominates the binding affinity. The bioassay result $\left(\mathrm{IC}_{50} 0.6 \mu \mathrm{M}, 5\right.$ vs. 2.0 $\mu \mathrm{M}, 6)$, however, is not so consistent with the docking score.
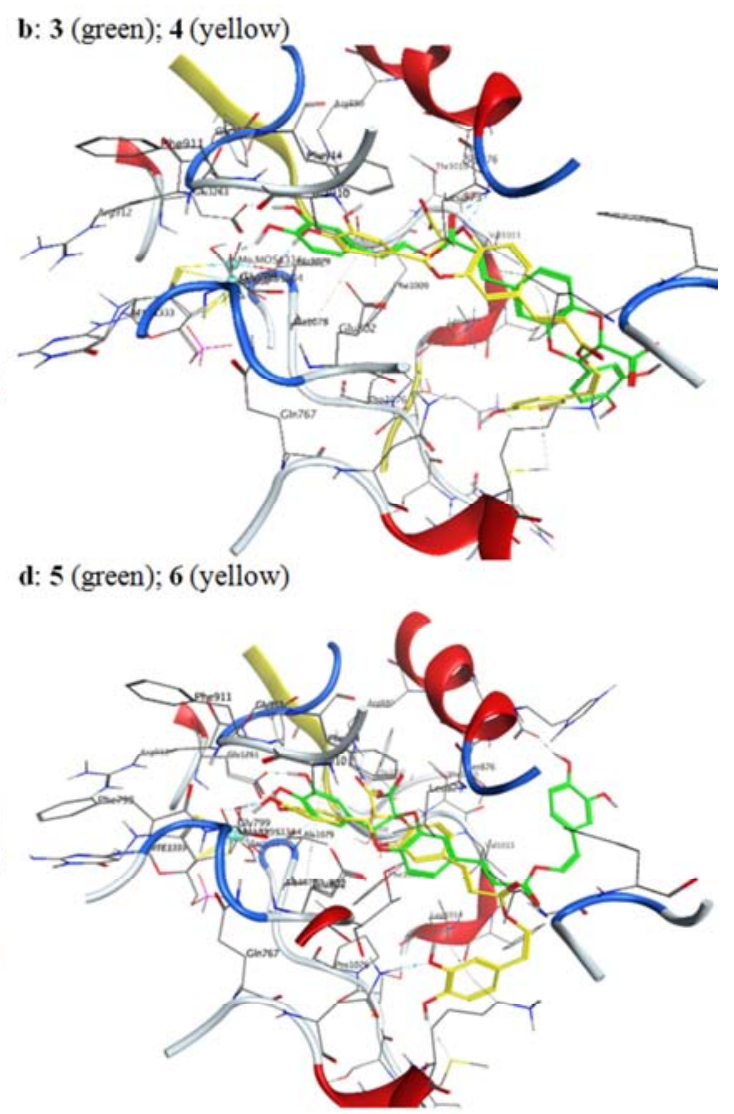

Figure 2. Molecular docking of 1 versus 2 (a), 3 versus 4 (b), 4 versus 6 (c), and 5 versus 6 (d) to XO template using the refinement. 
Attempts to verify this inconsistency were made and it was found that using the refinement (Forcefield-Affinity $\Delta G$ ) mode, not only the docking scores of 5 and 6 (Table 1) but also those of the rest compounds were in good agreement with the bioassay results. Thus this refinement mode is adopted for later on study.

Phlorotannins, rich in the seaweeds of the Ecklonia genus, such as E. cava and E. stolonifera, are polyphenolics like compounds $1-7$. Thus they might possess anti-XO activity. To support this assumption, molecular docking of some phlorotannins $(8-15)$ (Figure 3) $[6,7]$ to XO was undertaken via the London-refinement (Forcefield-Affinity $\Delta G$ ) mode. The result indicated that phlorofucofuroeckol B (11), 2,7-phloroglucinol-6,6-bieckol and pyrogallol-phloroglucinol-6,6-bieckol (15) showed better affinity with the docking score in the range from -23.23 to $-24.07 \mathrm{Kcal} / \mathrm{mol}$. This result correlated well with the bioassay data of three available phlorotannins, 2-phloroeckol (9), 6,6'-bieckol (12) [14], and dieckol (13) [15], against xanthine oxidase (Table 2). The $\mathrm{IC}_{50}$ values of these three compounds ranged from $5.6 \mu \mathrm{M}(13)$ to $18.2 \mu \mathrm{M}(9)$, comparable to that of the positive control allopurinol $\left(\mathrm{IC}_{50} 8.5 \mu \mathrm{M}\right)$. While using the refinement (Forcefield-London) mode, the docking and the bioassay results of these three compounds (Table 2) were not consistent as that described in 5 and 6 .

Since the pocket of the active site is another choice for molecular docking and the alpha mode is another refinement method, they were applied to virtually screen these three available phlorotannins. The result indicated the corresponding conformation for the best docking score was seriously distorted for the planar phenyl group. Thus the alpha refinement mode and the docking on the pocket are not suitable as virtual screening tool for this type compounds.

\subsection{Interaction of XO with Phlorotannins 8-15}

Compound 9 is the 2-O-phloroglucinolated derivative of eckol (8). This additional substitution in 9 caused steric hindrance to the binding site, leading to two H-bonds less than 8 and hence weaker binding affinity than $8(\Delta G-18.16$ vs. $-20.99 \mathrm{Kcal} / \mathrm{mol}$ ) (Figure 4a).

For the structural isomers of di-phloroglucinolated eckols 10 and 11 , the conformation of 11 is linear-like while that of 10 contains an L-shape skeleton to give a skew conformation with two phloroglucinol residues. Such difference allowed 11 to have higher flexibility to let it adapt much better to the binding pocket than 10 , reflected by stronger binding affinity $(\Delta G-24.07$ vs. $-20.31 \mathrm{Kcal} / \mathrm{mol})$.<smiles>[R]Oc1cc(O)c2c(c1[R])Oc1c(O)cc(O)cc1O2</smiles>

$\mathbf{8} \mathrm{R}^{\prime}=\mathbf{A}, \mathrm{R}^{\prime \prime}=\mathrm{H} ; \mathrm{MF} \mathrm{C}_{18} \mathrm{H}_{12} \mathrm{O}_{9} 372.28$ $9 \mathrm{R}^{\prime}=\mathbf{A}, \mathrm{R}^{\prime \prime}=\mathbf{B} ; \mathrm{MF} \mathrm{C}_{24} \mathrm{H}_{16} \mathrm{O}_{12} 496.38$<smiles>[R]c1c(O)cc(O)c2c1oc1c(O)cc(O)cc1oc1c(O)cc(O)c([R])c12</smiles>

$10 \mathrm{R}=\mathbf{A} ; \mathrm{MF} \mathrm{C}_{30} \mathrm{H}_{18} \mathrm{O}_{14} 602.46$<smiles>[R]c1cc(O)c2oc3c(O)c(O)cc(O)c3c3c([R])c(OC)cc(O)c3oc2c1[R]</smiles>

$14 \mathrm{R}^{\prime}=\mathbf{A}, \mathrm{R}^{\prime \prime}=\mathbf{B} ; \mathrm{MF} \mathrm{C}_{48} \mathrm{H}_{30} \mathrm{O}_{23} 974.74$<smiles>[R]c1c(O)cc(O)c2c1Oc1c(cc3oc4c([R])c(O)cc(O)c4c3c1O)O2</smiles>

$11 \mathrm{R}=\mathbf{A} ; \mathrm{MF} \mathrm{C}_{30} \mathrm{H}_{18} \mathrm{O}_{14} 602.46$<smiles></smiles>

$12 \mathrm{R}=\mathbf{A} ; \mathrm{MF} \mathrm{C}_{36} \mathrm{H}_{22} \mathrm{O}_{18} 742.55$<smiles>[Z7]c1c(O)cc(O)c2c1Oc1c(O)cc(O)c(-c3c(O)cc(O)c4c3Oc3c(O)cc(O)c([Z7])c3O4)c1O2</smiles>

$15 \mathrm{R}^{\prime}=\mathbf{A}, \mathrm{R}^{\prime \prime}=\mathbf{C} ; \mathrm{MF} \mathrm{C}_{48} \mathrm{H}_{30} \mathrm{O}_{23} 974.74$<smiles>Oc1cc(O)cc(O)c1</smiles><smiles>Oc1cc(O)c(I)c(O)c1</smiles><smiles>[R]c1c(O)cc(O)c2c1Oc1c(O)cc(Oc3c(O)cc(Oc4c(O)cc(O)c5c4Oc4c(O)cc(O)cc4O5)cc3O)cc1O2</smiles>

$13 \mathrm{R}=\mathbf{A} ; \mathrm{MF} \mathrm{C}_{36} \mathrm{H}_{22} \mathrm{O}_{18} 742.55$<smiles>CCOc1cc(O)cc(Oc2cc(O)c(Oc3cc(O)cc(O)c3)c(O)c2)c1</smiles>

C

Figure 3. Structures of phlorotannins 8-15. 

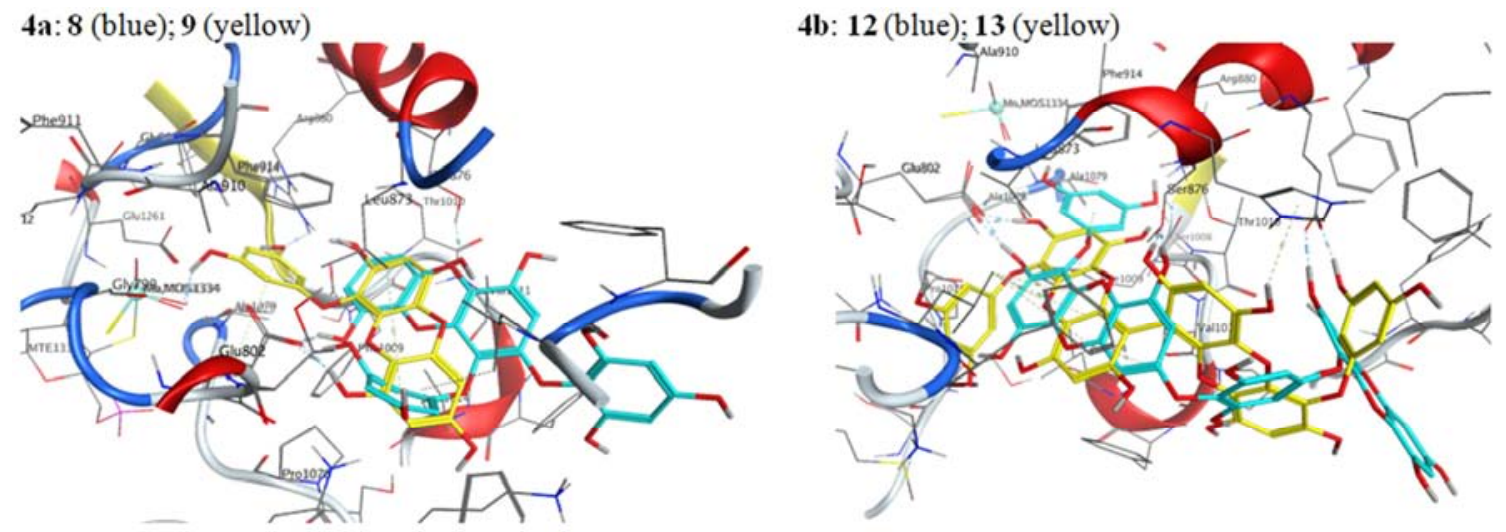

Figure 4. Molecular docking of 8 versus 9 (4a) and 12 versus 13 (4b) to xanthine oxidase template.

Table 1. IC 50 of seven oligo-phenylpropanoids against xanthine oxidase and their binding affinity, based on molecular docking (refinement: Forcefield-London / Forcefield-Affinity $\Delta G$ )

\begin{tabular}{|c|c|c|c|c|c|c|c|c|c|c|}
\hline \multirow{3}{*}{ Compd } & \multirow{3}{*}{$\mathrm{IC}_{50}{ }^{a}(\mu \mathrm{M})$} & \multirow{3}{*}{$\Delta G_{\text {calc }} \mathrm{kcal} / \mathrm{mol}$} & \multicolumn{8}{|c|}{ Number of Ligand-Receptor Interaction } \\
\hline & & & \multicolumn{2}{|c|}{ H-bond } & \multicolumn{2}{|l|}{$n-\pi$} & \multicolumn{2}{|l|}{$\pi-\pi$} & \multicolumn{2}{|c|}{ ionic } \\
\hline & & & $\mathbf{T}^{b}$ & $\mathbf{A}^{c}$ & $\mathbf{T}$ & $\mathbf{A}$ & $\mathbf{T}$ & $\mathbf{A}$ & $\mathbf{T}$ & $\mathbf{A}$ \\
\hline 1 & $>159.2$ & $-15.33 /-15.66$ & $1 / 2$ & $0 / 1$ & $0 / 1$ & $0 / 1$ & $1 / 1$ & $1 / 1$ & $0 / 0$ & $0 / 0$ \\
\hline 2 & $11.7 \pm 2.5$ & $-19.32 /-19.11$ & $4 / 4$ & $2 / 2$ & $1 / 1$ & $1 / 1$ & $1 / 1$ & $1 / 1$ & $0 / 0$ & $0 / 0$ \\
\hline 3 & $39.5 \pm 6.6$ & $-17.83 /-17.78$ & $4 / 3$ & $2 / 1$ & $2 / 0$ & $1 / 0$ & $1 / 1$ & $1 / 1$ & $0 / 0$ & $0 / 0$ \\
\hline 4 & $5.2 \pm 0.5$ & $-20.86 /-20.89$ & $3 / 2$ & $3 / 2$ & $3 / 1$ & $1 / 0$ & $1 / 1$ & $1 / 1$ & $0 / 4$ & $0 / 4$ \\
\hline 5 & $0.6 \pm 0.3$ & $-21.95 /-21.84$ & $4 / 4$ & $2 / 2$ & $0 / 1$ & $0 / 1$ & $1 / 1$ & $1 / 1$ & $4 / 4$ & $4 / 4$ \\
\hline 7 & $30.9 \pm 0.8$ & $-17.87 /-17.58$ & $4 / 1$ & $2 / 0$ & $2 / 1$ & $2 / 0$ & $0 / 0$ & $0 / 0$ & $0 / 0$ & $0 / 0$ \\
\hline allopurinol & $5.3 \pm 0.6$ & $-11.12 /-11.18$ & $3 / 3$ & $3 / 3$ & $1 / 1$ & $1 / 1$ & $1 / 1$ & $1 / 1$ & $0 / 0$ & $0 / 0$ \\
\hline
\end{tabular}

${ }^{a}$ Data adopted from [5]; ${ }^{b}$ total interaction number; ${ }^{c}$ interaction number at the active site.

Table 2. Binding affinity of eight phlorotannins (8-15) toward xanthine oxidase based on molecular docking (refinement: Forcefield-London / Forcefield-Affinity $\Delta G$ ) and the $I C_{50}$ of 9,12 , and 13 against xanthine oxidase.

\begin{tabular}{|c|c|c|c|c|c|c|c|c|c|c|}
\hline \multirow{3}{*}{$\operatorname{Compd}^{a}$} & \multirow{3}{*}{$\mathrm{IC}_{50}(\mu \mathrm{M})$} & \multirow{3}{*}{$\begin{array}{l}\Delta G_{\text {calc }}{ }^{*} \\
(\mathrm{kcal} / \mathrm{mol})\end{array}$} & \multicolumn{8}{|c|}{ Number of Ligand-Receptor Interaction* } \\
\hline & & & \multicolumn{2}{|c|}{ H-bond } & \multicolumn{2}{|l|}{$\mathbf{n}-\pi$} & \multicolumn{2}{|l|}{$\pi-\pi$} & \multicolumn{2}{|c|}{ ionic } \\
\hline & & & $\mathbf{T}^{b}$ & $\mathbf{A}^{c}$ & $\mathbf{T}$ & $\mathbf{A}$ & $\mathbf{T}$ & $\mathbf{A}$ & $\mathbf{T}$ & $\mathbf{A}$ \\
\hline 8 & - & $-20.70 /-20.36$ & $5 / 4$ & $3 / 3$ & 0 & 0 & $1 / 1$ & $1 / 1$ & $0 / 0$ & $0 / 0$ \\
\hline 9 & $18.20 \pm 1.70$ & $-22.13 /-18.16$ & $4 / 3$ & $3 / 1$ & $1 / 3$ & $1 / 2$ & $1 / 0$ & $1 / 0$ & $0 / 0$ & $0 / 0$ \\
\hline 10 & - & $-19.49 /-20.31$ & $2 / 2$ & $2 / 2$ & $7 / 3$ & $3 / 1$ & $1 / 1$ & $1 / 1$ & $0 / 0$ & $0 / 0$ \\
\hline 11 & - & $-24.45 /-24.07$ & $5 / 6$ & $4 / 4$ & $4 / 3$ & $3 / 2$ & $1 / 2$ & $1 / 2$ & $0 / 0$ & $0 / 0$ \\
\hline 12 & $7.40 \pm 1.20$ & $-17.95 /-19.27$ & $3 / 3$ & $2 / 2$ & $4 / 3$ & $2 / 1$ & $0 / 0$ & $0 / 0$ & $0 / 0$ & $0 / 0$ \\
\hline 14 & - & $-20.16 /-23.75$ & $6 / 7$ & $3 / 4$ & $2 / 5$ & $1 / 2$ & $0 / 0$ & $0 / 0$ & $0 / 0$ & $0 / 0$ \\
\hline 15 & - & $-24.27 /-23.23$ & $4 / 5$ & $3 / 3$ & $6 / 4$ & $1 / 1$ & $1 / 0$ & $1 / 0$ & $0 / 0$ & $0 / 0$ \\
\hline allopurinol & $8.50 \pm 0.70$ & $-11.12 /-11.18$ & $3 / 3$ & $3 / 3$ & $1 / 1$ & $1 / 1$ & $1 / 1$ & $1 / 1$ & $0 / 0$ & $0 / 0$ \\
\hline
\end{tabular}

*: Forcefield-London / Forcefield-Affinity $\Delta G ;{ }^{a}$ 2-phloroeckol (9), 6,6'-bieckol (12), dieckol (13); ${ }^{b}$ total interaction number; ${ }^{c}$ interaction number at the active site.

Similarly, for the isomeric dieckols 12 and 13, the conformation of 13 is more extended and flexible than that of 12 (Figure $4 \mathrm{~b}$ ) and thus 13 had stronger binding affinity $(\Delta G$ -20.99 vs. $-19.27 \mathrm{Kcal} / \mathrm{mol}$ ), being consistent with the bioassay result $\left(\mathrm{IC}_{50} 5.6 \mu \mathrm{M}, 13\right.$ vs. $\left.7.4 \mu \mathrm{M}, 12\right)$. (Figure $4 \mathrm{~b}$ ) and thus had stronger binding affinity $(\Delta G-20.99$ vs. -19.27 $\mathrm{Kcal} / \mathrm{mol}$ ), being consistent with the bioassay result $\left(\mathrm{IC}_{50} 5.6\right.$ $\mu \mathrm{M}, 13$ vs. $7.4 \mu \mathrm{M}, 12$ ).

Compounds 14 and 15 are di-phloroglucinolated 6,6'-bieckols and are positional isomers. The visual comparison showed that the structure conformation of 14 was relatively bulky than that of 15 . While insertion of the bottom bulky phloroglucinol residue of 14 into the binding site blocked the binding pocket entrance, accounting for a little higher binding score $(\Delta G-23.75 \mathrm{Kcal} / \mathrm{mol}, 14$ vs. -23.23 $\mathrm{Kcal} / \mathrm{mol}, 15$ ).

As phlorofucofuroeckol B (11), 6,6-bieckol of 2,7-phloroglucinol (14), and pyrogallol-phloroglucinol (15) showed better affinity to xanthine oxidase than dieckol (13), they might be more potent XO inhibitors and are worthy of further investigation if they are available.

\section{Conclusion}

The binding affinity of compounds (1-15) toward xanthine oxidase showed that the interaction associated strongly with 
certain amino acid residues in the active site such as Arg880, Thr1010, and Glu1261. These amino acid residues form $\mathrm{H}$-bond or/and ionic interaction with the active ligands, which play critical roles against XO. Using the refinement (Forcefield-Affinity $\Delta G$ ) mode, the docking results of either phenylpropanoids $1-7$ or three phlorotannins $(9,12,13)$ were more consistent with the bioassay results than those obtained by the refinement (Forcefield-London) mode. Thus the London (trimatch)-refinement (Forcefield-Affinity $\Delta G$ ) docking method is recommended for virtual screening of the related phenolic compounds. As 6,6'-bieckol (12) and dieckol (13) showed inhibitory activity against XO comparable to the positive control allopurinol, three phlorotannins $(11,14,15)$ having better docking score than 12 and 13 deserve further study. Since these phlorotannins are rich in Ecklonia cava and E. stolonifera, these common edible seaweeds should be beneficial to persons suffering hyperuricemia.

\section{Acknowledgements}

We gratefully acknowledge the financial support from Ministry of Science and Technology, Taiwan, ROC under the grant MOST 103-2320-B-002-011-MY3.

\section{References}

[1] R. Hille (2006) Structure and function of xanthine oxidoreductase. European Journal of Inorganic Chemistry 10: 1913-1926.

[2] Rock, K. L., H. Kataoka, J. J. Lai (2013) Uric acid as a danger signal in gout and its comorbidities. Nature Reviews Rheumatology 9: 13-23.

[3] Pacher, P., A. Nivorozhkin, C. Szabo (2006) Therapeutic effects of xanthine oxidase inhibitors: renaissance half a century after the discovery of allopurinol. Pharmacology Review 58: 87-114.

[4] Robinson, P. C., N. Dalbeth (2015) Advances in pharmacotherapy for the treatment of gout. Expert Opinion on Pharmacotherapy 16: 533-546.
[5] Tsai, S. F., S. S. Lee (2014) Neolignans as xanthine oxidase inhibitors from Hyptis rhomboids. Phytochemistry 101: 121127.

[6] Enroth, C., B. T. Eger, K. Okamoto, T. Nishino, T. Nishino, E. F. Pai (2000) Crystal structures of bovine milk xanthine dehydrogenase and xanthine oxidase: structure-based mechanism of conversion. Proceedings of the National Academy of Sciences USA 97: 10723-10728.

[7] Molecular Operating Environment (MOE) 2010.10. (2010). Chemical Computing Group, Inc; Montreal, Quebec.

[8] Herbert Edelsbrunner, Ernst P. Mücke (1992) Three-dimensional alpha shapes. Mathematics, Computer Science VVS: $75-82$.

[9] Matteo Aldeghi, Alexander Heifetz, Michael J. Bodkin, Stefan Knapp and Philip C. Biggin (2016) Accurate calculation of the absolute free energy of binding for drug molecules. Chemical Science 7: 207-218.

[10] María J. R. Yunta (2016) Docking and Ligand Binding Affinity: Uses and Pitfalls. American Journal of Modeling and Optimization 4 (3): 74-114.

[11] T. A. Halgren (1996) Merck molecular force field. 1. Basis, form, scope, parameterization, and performance of MMFF94. Journal of Computational Chemistry 17: 490-519.

[12] Clark AM, Labute P. (2007) 2D depiction of protein-ligand complexes. J Chem Inf Model. 47 (5): 1933-1944.

[13] Hsu, F. C., S. F. Tsai, S. S. Lee (2019) Chemical investigation of Hyptis suaveolens seed, a potential antihyperuricemic nutraceutical, with assistance of HPLC-SPE-NMR. Journal of Food and Drug Analysis 27: 897-905 and reference cited therein.

[14] Lee, M. S., T. Shin, T. Utsuki, J. S. Choi, D. S. Byun, H. Kim (2012) Isolation and identification of phlorotannins from Ecklonia stolonifera with antioxidant and hepatoprotective properties in tacrine-treated HepG2 cells. Journal of Agriculture and Food Chemistry 60: 5340-5349.

[15] Lee, J. H., J. Y. Ko, J. Y. Oh, C. Y. Kim, H. J. Lee, J. Kim, Y. J. Jeon (2014) Preparative isolation and purification of phlorotannins from Ecklonia cava using centrifugal partition chromatography by one-step. Food Chemistry 158: 433-437. 